\title{
Neuer Lehrgang mit optionalem E-Learning in medizinischem Strahlenschutz für Ärztinnen und Ärzte
}

\author{
Marc Otto , \\ Jean-François Valley ${ }^{b}$ \\ Max Giger ${ }^{\circ}$ \\ a pnn ag, Zürich \\ b ehem. Leiter IRA, Lausanne \\ c Präsident KWFB, Mitglied \\ des Zentralvorstandes der FMH
}

\section{Zusammenfassung}

Aufgrund der Erfahrungen aus den Strahlenschutzkursen für Ärztinnen und Ärzte, die zwischen 1994 und 2004 erteilt worden waren, haben sich das Bundesamt für Gesundheit (BAG) und die Verbindung der Schweizer Ärztinnen und Ärzte (FMH) auf eine neue Gewichtung und Vereinheitlichung der Lernziele geeinigt. Die notwendigen Inhalte für Sachkunde und Sachverstand werden in Zukunft allen radiologisch tätigen Ärztinnen und Ärzten vermittelt, unabhängig davon, ob sie innerhalb einer Institution oder selbständig arbeiten. Die theoretischen Inhalte der Strahlenschutzweiterbildung können in Präsenzkursen oder mittels eines E-Learning-Kurses, die praktischen Inhalte im Labor bzw. an Modellen erworben werden. Entsprechend dem angestrebten Facharzttitel erfolgt eine stärkere Gewichtung von Durchleuchtung oder Röntgenaufnahmen. Diese Weiterbildung wird mittels einer einheitlichen Multiple-choice-Prüfung abgeschlossen. Durch die Schaffung eines E-Learning-Kurses kann die physische Präsenz auf zwei Tage reduziert werden. Eine neue zentrale Anmeldeplattform (www.radioprotection.ch) verschafft Übersicht auf das gesamte Kursangebot der Schweiz und ermöglicht Ärztinnen und Ärzten, sich online zum Kurs der Wahl einzuschreiben.

Die Vermittlung der klinischen bzw. fachspezifischen Lerninhalte bleibt weiterhin Sache der von der FMH für die ärztliche Weiterbildung anerkannten Weiterbildungsstätten.

\section{Einleitung}

Die Ärzteschaft (FMH) und die Aufsichtsbehörde (BAG) haben sich auf die Durchführung eines gemeinsamen Projekts geeinigt, um die Theorie des Strahlenschutzes via E-Learning anzubieten. Mit der Entwicklung der Inhalte beauftragte das BAG einen Medizinphysiker mit Erfahrung im Strahlenschutz und in der Röntgentechnik. Für die Umsetzung wurde die pnn ag, ein Spin-off-Unternehmen der ETH Zürich, beauftragt. Zur Projektsteuerung, namentlich zur Entwicklung und Pflege der Inhalte wurde ein Beirat geschaffen. Dieser setzte sich aus je einem Vertreter der
Schweizerischen Gesellschaft für medizinische Radiologie und der Schweizerischen Gesellschaft für Allgemeinmedizin sowie einem Medizinphysiker zusammen. Als ständige Gäste wurden FMH und BAG eingeladen. Die Gewichtung und Zuordnung der Lernziele wurden von einem weiteren Gremium festgelegt, in dem neben Radiologie, Allgemeinmedizin und Medizinphysik auch der Verband der Schweizer Assistenz- und Oberärztinnen und -ärzte (VSAO) sowie BAG und FMH vertreten waren.

\section{Lerninhalt}

Der theoretische und praktische Lerninhalt wurden aufgrund der Strahlenschutzgesetzgebung aufgestellt und in vier theoretische Teile und einen praktischen Teil gegliedert.

\section{Grundlagen des Strahlenschutzes}

- Ionisierende Strahlen;

- Wechselwirkung zwischen Strahlung und Materie;

- biologische Wirkungen;

- Grundsätze des Strahlenschutzes.

\section{Röntgentechnik}

- Funktionsweise einer Röntgenanlage;

- Wechselwirkung zwischen Strahlung und Patient;

- Aufzeichnung der Strahlung;

- Betriebsarten von radiologischen Anlagen.

\section{Schutzmassnahmen bei Anwendung von Röntgenstrahlen}

- Bestimmung der Patientendosis;

- Bildqualität in der Radiologie;

- Optimierung der Patientendosis;

- Schutz der beruflich strahlenexponierten Personen.

\section{Strahlenschutzgesetzgebung}

- Gesetzgebung im Strahlenschutz;

- Bewilligungspflicht und Aufgaben des Sachverständigen;

- Qualitätskontrollen in der Röntgendiagnostik;

- diagnostische Referenzwerte. 


\section{Praktikum}

- Funktionieren der Röntgenanlagen;

- Patientendosimetrie und Einstelltechnik;

- praktische Methoden zur Minimierung der Strahlenexposition des Patienten und des Operateurs.

\section{Kontrollierte Onlineprüfung}

Da somit, quasi als Nebenprodukt des E-Learnings, ein detaillierter Lernzielkatalog entstanden ist, haben die vom BAG anerkannten Ausbildungsstätten in medizinischem Strahlenschutz für Ärzte beschlossen, als Abschluss ein einheitliches Prüfungsverfahren einzusetzen. Zur Überwachung dieser Prüfung und zur Pflege des Fragenpools wurde eine Prüfungskommission eingesetzt, in der jede Ausbildungsstätte mit einem Vertreter Einsitz hat.

Aufgrund des Lernzielkataloges wurden ein Blueprint und ein Pool von Multiple-choice-Fragen entwickelt. An der Prüfung wird für jeden Kandidaten eine individuelle Auswahl von Fragen zusammengestellt, wobei der Blueprint dafür sorgt, dass die Gewichtung der geprüften Lernziele dieselbe bleibt und dass auch die Anteile der verwendeten Fragetypen gleichbleiben.

An der Prüfung teilnehmen kann jeder Kandidat, der sowohl Theorie wie Praxis erfolgreich besucht hat. Die Prüfung besteht aus 32 Multiple-choice-Fragen $(\mathrm{A}+, \mathrm{A}-, \mathrm{Kprim})$, die innert 60 Minuten beantwortet werden müssen. Die Prüfung gilt als bestanden, wenn mindestens 21 Fragen korrekt beantwortet worden sind. Bei Nichtbestehen kann die Prüfung wiederholt werden. Als Hilfsmittel während der Prüfung sind ausschliesslich die rechtlichen Grundlagen (Strahlenschutzgesetz und zugehörige Verordnungen) zugelassen.

Bis jetzt wurden die Prüfungen gleich im Anschluss ans Praktikum in einem Computerraum durchgeführt. Da es bei Onlineprüfungen unmöglich ist, die Kandidaten auf vollautomati-

\footnotetext{
Vereinfachte Anmeldung unter www.radioprotection.ch

Um den Ärztinnen und Ärzten den Einschreibeprozess zu einem Strahlenschutzkurs zu vereinfachen, haben die vier anerkannten Ausbildungsstätten, Paul Scherrer Institut PSI, Institut de radiophysique appliquée IRA, SAFPRO und pnn ag, eine gemeinsame Internetplattform (www.radioprotection.ch) etabliert. Dort sind sämtliche Strahlenschutzkurse als vollständige Kurspakete mit Theorie, Praxis und Abschlussprüfung, alle zugehörigen Informationen (Ort, Zeiten, Kosten) sowie die noch verfügbaren Plätze aufgeführt. Sobald die entsprechende Ausbildungsstätte eine Anmeldung validiert hat, wird via E-Mail die entsprechende Kursbestätigung ausgestellt. Von da an sind für jeden Teilnehmer seine individuellen Kursinformationen passwortgeschützt abrufbar.
}

schem Weg zuverlässig zu identifizieren, muss die organisierende Ausbildungsstätte bestätigen, dass tatsächlich der angemeldete Kandidat die Prüfung am PC ablegt. Nach Abschluss wird die Prüfung sofort korrigiert und ausgewertet. Das BAG beauftragte die pnn ag mit der Durchführung dieser Onlineprüfung.

\section{Erste Erfahrungen mit neuer Struktur des Lehrgangs}

Bis Ende August wurden in der Schweiz bereits 14 Lehrgänge von über 140 Ärztinnen und Ärzten besucht, wobei sich über $40 \%$ bereits über die gemeinsame Onlineplattform www. radioprotection.ch eingeschrieben hatten. Sämtliche Prüfungen konnten über das neue Onlinesystem abgewickelt und die entsprechenden Abschlusszeugnisse (Verfügungen) ausgestellt werden.

Die Straffung und Vereinheitlichung des Lernzielkatalogs sowie der Abschluss des Kurses mit einer strukturierten Prüfung hat die Anforderungen sowohl für die Ausbildungsstätten als auch für die Auszubildenden erhöht.

Da insbesondere der Theorieteil eine geballte Ladung Stoff vermittelt, wird vor allem bei Kursen, die an vier aufeinanderfolgenden Tagen stattfinden, mit einem neuen Kursablauf experimentiert, bei dem kürzere Theorieblöcke alternierend mit Praktika kombiniert werden. So sollen die Theorieeinheiten kleiner und der Kurs abwechslungsreicher werden.

Beim E-Learning wurden neben kleineren Anpassungen im Bereich der Benutzerführung vor allem die Elemente zur Repetition des Stoffes und zur Prüfungsvorbereitung ausgebaut.

Die Prüfungskommission musste bis jetzt noch keinerlei Rekurse behandeln. In der Anlaufphase des neuen Kurskonzeptes werden sämtliche nicht bestandenen Prüfungen einzeln von Experten überprüft. Zudem werden alle eingesetzten Fragen aufgrund einer statistischen Auswertung der in Prüfungen gegebenen Antworten analysiert und in Sitzungen der Prüfungskommission besprochen. So hat die Prüfungskommission den Fragepool in einer ersten Sitzung bereits verfeinert.

\section{E-Learning}

Das E-Learning basiert auf einer detaillierten, konsequent umgesetzten Struktur, die den Kurs in 16 Lektionen mit jeweils 10 Kapiteln unterteilt. Jedes der 160 Kapitel behandelt spezifische Lernziele. Dem Kurstyp (Röntgenaufnahme, Durchleuchtung) entsprechend werden die Kapitel automatisch konfektioniert. Für das Testat, das den erfolgreichen Besuch des E-Learning- 


\begin{abstract}
Aktueller Lehrgang medizinischer Strahlenschutz für Ärzte
Seit Anfang 2007 werden die neuen Strahlenschutzkurse für Ärzte durchgeführt. Das erfolgreiche Absolvieren dieser Kurse ist obligatorisch sowohl für Ärztinnen und Ärzte, die ein Bewilligungsgesuch zum Betreiben von Röntgenanlagen einreichen wollen, als auch für diejenigen, die einen Facharzttitel anstreben, dessen Weiterbildungsprogramm eine Weiterbildung in Strahlenschutz verlangt.

Der Strahlenschutzkurs wird in zwei Varianten angeboten: konventionelle Aufnahmetechnik (Typ A) und Durchleuchtung (Тур B). Er besteht aus einem theoretischen und einem praktischen Teil im Umfang von je zwei Ausbildungstagen sowie einer abschliessenden standardisierten Multiple-choice-Prüfung. Der theoretische Teil kann entweder an zwei Kurstagen in traditioneller Form besucht oder via Internet als E-Learning unabhängig von Zeit und Ort absolviert werden. Der praktische Teil muss zwingend als Präsenzunterricht an einer anerkannten Ausbildungsstätte besucht werden, da dort mit Röntgengeräten gearbeitet und die MC-Prüfung an einem Computer absolviert wird.
\end{abstract}

Kurses bestätigt, müssen alle Pflichtkapitel bearbeitet worden sein. Dieses Testat ermöglicht, direkt die zwei Praktikatage zu besuchen und danach die Prüfung abzulegen. Die Abfolge, in der die 16 Lektionen bearbeitet werden müssen, ist teilweise vorgegeben. So stehen am Anfang die erste Lektion, die die physikalischen Grundlagen ionisierender Strahlung behandelt, sowie die vier Lektionen zur Strahlenschutzgesetzgebung offen. Die übrigen elf Lektionen, die die physikalischen Grundlagen, Röntgenanlagen, und die patientenorientierten Aspekte des medizinischen Strahlenschutzes behandeln, bauen aufeinander auf und müssen sequentiell bearbeitet werden.

Jede Lektion ist gleich aufgebaut und enthält dieselben Elemente: die Fragestellung (Lernziele), eine Zusammenfassung, die die Fragestellung beantwortet, sowie die ausführlichen Lerninhalte, die die Aussagen der jeweiligen Zusammenfassung detailliert behandeln. Die Lerninhalte können mit Übungen, Animationen und weiterführenden Informationen weiter vertieft werden. Zu jeder Lektion stehen mehrere Kontrollfragen zur Verfügung, um den Lernfortschritt zu überprüfen.

Um ein individuelles Lernerlebnis zu vermitteln, können die Lernenden aus drei verschiedenen Arten auswählen, wie sie (denselben) Lerninhalt zu bearbeiten wünschen. Diese unterscheiden sich jeweils in Auswahl und Abfolge der einzelnen Elemente. Im «Standardmodus» folgt pro Kapitel auf die Fragestellung der ausführliche Lerninhalt, eine Lernerfolgskontrollfrage und schliesslich - als Repetition - die Zusammenfassung. Im «Expressmodus» werden zuerst Fragestellung und Zusammenfassung angezeigt. Fühlen sich die Lernenden sicher genug, können sie den Lerninhalt überspringen und gleich zur Lernerfolgskontrolle gelangen. Wurde allerdings die Frage nicht korrekt beantwortet, gelangt man automatisch zum ausführlichen Lerninhalt. Bei beiden Modi gilt ein Kapitel als bearbeitet, sobald eine Kontrollfrage richtig beantwortet worden ist. Wurde eine Frage falsch beantwortet, so wird für den nächsten Versuch eine weitere Frage präsentiert. Für Lernende, die bereits über gute Vorkenntnisse verfügen, steht zu jeder Lektion auch ein Check-up bereit. Nachdem man dort die gewünschten Kapitel ausgewählt hat, werden pro Lektion zwei Kontrollfragen in einem Prüfungsmodus präsentiert. Wurden beide Fragen korrekt beantwortet, so gilt das Kapitel als bearbeitet. Für die Abschlussprüfung relevant sind jeweils nur die kurzen Zusammenfassungen der Pflichtkapitel.

\section{Pilotprojekt E-Learning}

Das E-Learning, das in deutscher und französischer Sprache gehalten ist, wurde nach Fertigstellung von einer Gruppe Assistenzärzte getestet. Diese Gruppe («peer group») wurde vom VSAO zusammengestellt und musste alle Kapitel durcharbeiten. $\mathrm{Zu}$ jedem Lernelement konnte online gleich ein Kommentar abgegeben werden. Zudem wurden nach Abschluss der Pilotphase qualitative Interviews geführt. Aufgrund der Kritik wurde das gesamte E-Learning noch einmal überprüft und Schwachstellen wurden behoben.

\section{Schlussfolgerungen}

Die theoretische Weiterbildung in Strahlenschutz wird zehn Jahre nach Inkraftsetzung des Strahlenschutzgesetzes unter Einsatz moderner Lehrmethoden effizienter gestaltet und den Bedürfnissen der Lernenden angepasst. Die praktische Weiterbildung, für die die Leiterinnen und Leiter der klinischen Weiterbildungsstätten verantwortlich sind, kann somit auf einer soliden Basis aufgebaut werden. 


\section{Auswertung der Peer-group-Interviews}

Peer-group

Die Peer-group bestand aus 7 Teilnehmern (4 Frauen, 3 Männer) im Alter zwischen 29 und 37 Jahren. Alle Teilnehmer mussten sämtliche 160 Kapitel des E-Learnings, also sowohl die Pflichtkapitel wie auch alle fakultativen, durcharbeiten, wofür sie im Durchschnitt 17 Stunden und 41 Minuten (Median) benötigten. Anschliessend wurden sie in Einzelgesprächen strukturiert befragt.

Resultate (technische Aspekte)
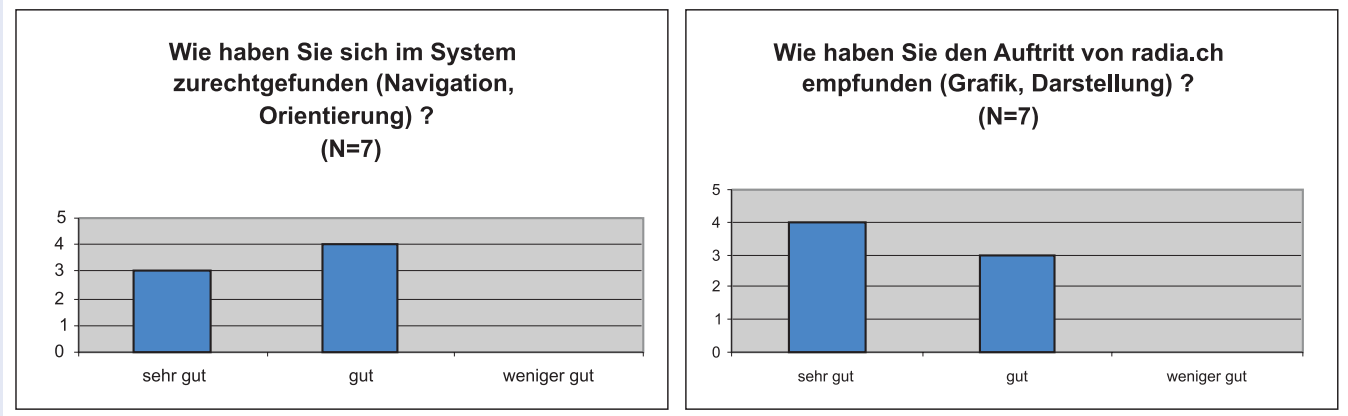

Positive Kommentare zum E-Learning

Zeitlich flexibles Bearbeiten möglich, eigenes Lerntempo, Selbstkontrolle durch Testfragen, guter Überblick, Repetitionsmöglichkeit, Animationen, guter Support durch pnn.

Kritische Kommentare zum E-Learning

Langsamer Seitenaufbau, Unklarheit bezüglich Prüfungsrelevanz.

Verbesserungsvorschläge, Erweiterungswünsche

Layout (dunklere Schrift, mehr Zeilenabstand, grössere Grafiken), Erinnerungs-E-Mails vor Prüfung, Zusammenfassung zum Ausdrucken, Tools zur Prüfungsvorbereitung.

Kritische Kommentare zur Strahlenschutzausbildung allgemein

- Balance zwischen Physik und Medizin;

- Komplexität der Materie;

- Umfang des vermittelten Stoffes. 